\title{
A Hybrid Microwave-Optical Applicator for Local Muscle Warming and Monitoring
}

\author{
Allann Al-Armaghany \\ Electronics and Electrical Engineering \\ University College London \\ London, United Kingdom \\ a.al-armaghany@ucl.ac.uk
}

\author{
Kin-Fai Tong \\ Electronics and Electrical Engineering \\ University College London \\ London, United Kingdom \\ k.tong@ucl.ac.uk
}

\author{
Terence Leung \\ Dept of Med Phys \& Biomedical Eng \\ University College London \\ London, United Kingdom \\ t.leung@ucl.ac.uk
}

\begin{abstract}
Local warming of human tissue causes vasodilation and therefore blood volume to increase. Such thermal blood volume response allows the assessment of microcirculation in tissue, providing clinically important information. The deep warming is provided by a novel microwave applicator, which has a annular-ring microstrip patch operating at $2450 \mathrm{MHz}$ with a superstrate interface layer to improve the coupling of electromagnetic (EM) waves into the skin. The design is based on computer simulations of specific absorption rate (SAR) and thermal distribution of the EM waves in a biological medium. The simulation results show that the applicator can elevate the muscle temperature by 3 to $4{ }^{\circ} \mathrm{C}$. The thermal response is measured by an integrated optical probe which measures tissue oxygenation changes in deep tissue using the near infrared spectroscopy technique.
\end{abstract}

\section{Keywords-Applicators, Antenna, SAR, NIRS}

\section{INTRODUCTION}

Microwave and radio frequency (RF) applicators have been employed in numerous therapeutic and diagnostic biomedical applications [1]. In cancer treatment, applicators operating in microwave and RF bands have been employed to induce heat in the cancerous tissue to increase the efficacy of radiotherapy and chemotherapy [2]. In physiotherapy, microwave applicators have also been used to aid muscle injury recovery [3]. In both cases, the temperature in the deep muscle is raised to approximately $41-45{ }^{\circ} \mathrm{C}$ [4]. Deposition of EM energy into the biological tissue is affected by several factors, including the proximity of the applicator, the dielectric properties of the biological tissue, and the variation of tissue thickness of each layer, such as skin, fat and muscle, among subjects [5].

Generally speaking, the applicator can be either noncontact or in direct contact with the patient's skin. For optimal selective warming, the applicator is best to operate in the near field region with direct contact where it can focus energy locally [6]. Direct contact applicators are more efficient in coupling the EM energy into the tissue and they can also reduce stray radiation [7]. Various ISM bands including 433, 915 and $2450 \mathrm{MHz}$ have been used. The frequency at $433 \mathrm{MHz}$ is popular in hyperthermia treatment due to an increase in penetration depth and its uniform SAR distribution [8], although the applicator's size is relatively large. Despite the reduction in penetration depth, an applicator at $2450 \mathrm{MHz}$ has been considered in this work, because it can deliver more localized heating and allows a compact design.

The aim of this work was to develop a device that can provide both warming and monitoring selectively in the deep tissue while minimizing any temperature change in the skin. This device can facilitate the investigation of thermoregulation in the deep tissue such as the muscle. The warming was performed by a novel microwave applicator, capable of elevating the local temperature in the muscle by 3 to $4^{\circ} \mathrm{C}$. The blood volume monitoring was performed by near infrared spectroscopy (NIRS) which has been widely employed in measuring haemoglobin concentration changes, a measure proportional to blood volume changes in deep tissues [9].

\section{MATERIAL AND METHOD}

\section{A. Microwave-optical applicator design}

The development of the hybrid microwave-optical probe focused on the integration of the optical emitter and detector with the microstrip patch antenna. Figure 1 shows a customized annular-ring patch applicator that was adopted which had matching electrical properties to be placed on a biological tissue. Coaxial probe feed method was selected to accommodate the optical emitter and detector in the confined space of the compact applicator. The applicator was in direct contact with the skin and its efficiency in inducing heat depended on the thicknesses of the skin, fat and muscle layers. A superstrate layer (Rogers RT/Duroid $6035 \mathrm{HTC}, \varepsilon_{\mathrm{r}}=3.5, \tan \delta=0.0013$, thermal conductivity $=$ $1.44 \mathrm{~W} / \mathrm{m} / \mathrm{K}$ and thickness $=2 \mathrm{~mm}$ ) was introduced between the skin and the applicator, acting as a match interface to provide a uniform field distribution, decrease stray radiation and minimize the applicator's size [10][11]

Figure 2 shows the configuration of the hybrid probe. Two types of optical monitoring were incorporated. The first was a near infrared spectroscopy (NIRS) probe which has been used widely to monitor oxygenation in deep tissues such as the muscle and the brain [9]. The optical emitter and detector were located on the radiating edges of the annular-ring microstrip patch applicator. In this work, an in-house built NIRS monitor was used which measured tissue oxygenation in terms of oxygenated blood $(\Delta \mathrm{HbO} 2)$, de-oxygenated bloody $(\Delta \mathrm{HHb})$ and total haemoglobin concentration changes $(\Delta \mathrm{HbT})$ with a single detector.

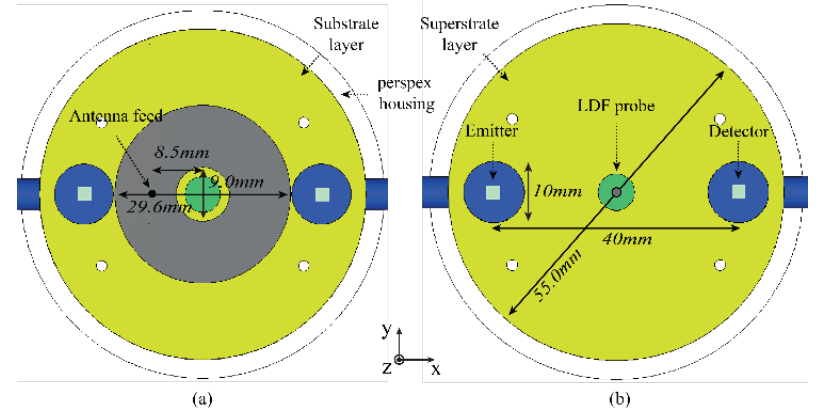


Fig. 1. Annular ring antenna design and optical sensors integration.

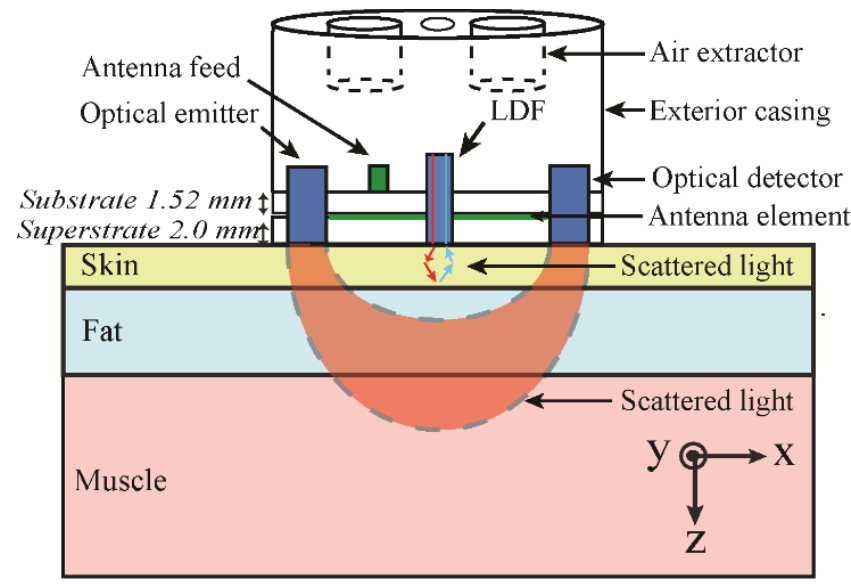

Fig. 2. Hybrid Applicator model

In conjunction to NIRS a commercial Laser Doppler blood flow (LDF) optical monitoring unit (moorVMS-LDF1, Moor Instruments) was used to measure the blood flow on the skin. The skin blood flow was monitored mainly to show that the skin temperature was kept stable during the microwave warming periods.

\section{B. Applicator Design based on computer simulation}

The main design parameters of the microwave applicator, including the type of material and dimension of the superstrate, substrate and antenna element, were determined and optimized based on the results from computer simulations. The simulations were performed CST Microwave Studio 2013. The software supports 3D meshes of a human calf (Voxel human model) in the simulations. The meshes consist of four layers, namely, the skin, fat, muscle and bone layers. Their electrical, physical, thermal and physiological properties are based on the voxel models.

\section{1) Specific Absorption Rate (SAR)}

Based on the applicator design, the corresponding EM radiation and the SAR distribution in a lossy medium (biological tissues) were simulated. In this study, the simulated SAR distribution was averaged over 1 gram of tissue. Fig. 3 shows the SAR distribution inside the calf model, as induced by the EM wave from the applicator in direct contact. The results were based on a $0.5 \mathrm{~W}$ power at the input port of the microstrip patch antenna, and the scale of the SAR value was normalized to a maximum value of $37.7 \mathrm{~W} / \mathrm{kg}$. Fig. 3 (a) depicts the SAR distribution in the $x z$ plane. High SAR values can be found in two localized regions at the skin-fat boundary. This indicates that the applicator was operating in its near-field region and the two local spots were caused by the fringing field at the radiating edges of the microstrip patch antenna in close proximity. Fig. 3(b) shows the SAR distribution in the $y z$-plane, the peak SAR was approximately $14 \mathrm{~mm}$ from the skin surface, which demonstrates the half peak power was deposited inside the muscle.
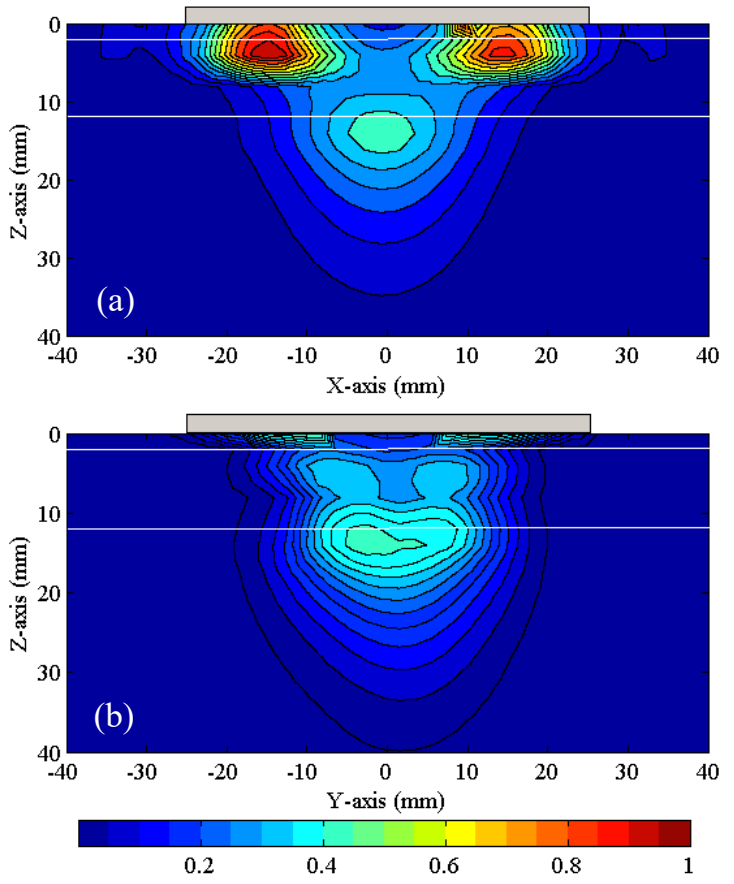

Fig. 3. Simulated SAR distribution with anatomical model (a) normalized SAR In $x z$-plane, (b) normalized SAR in $y z$-plane.
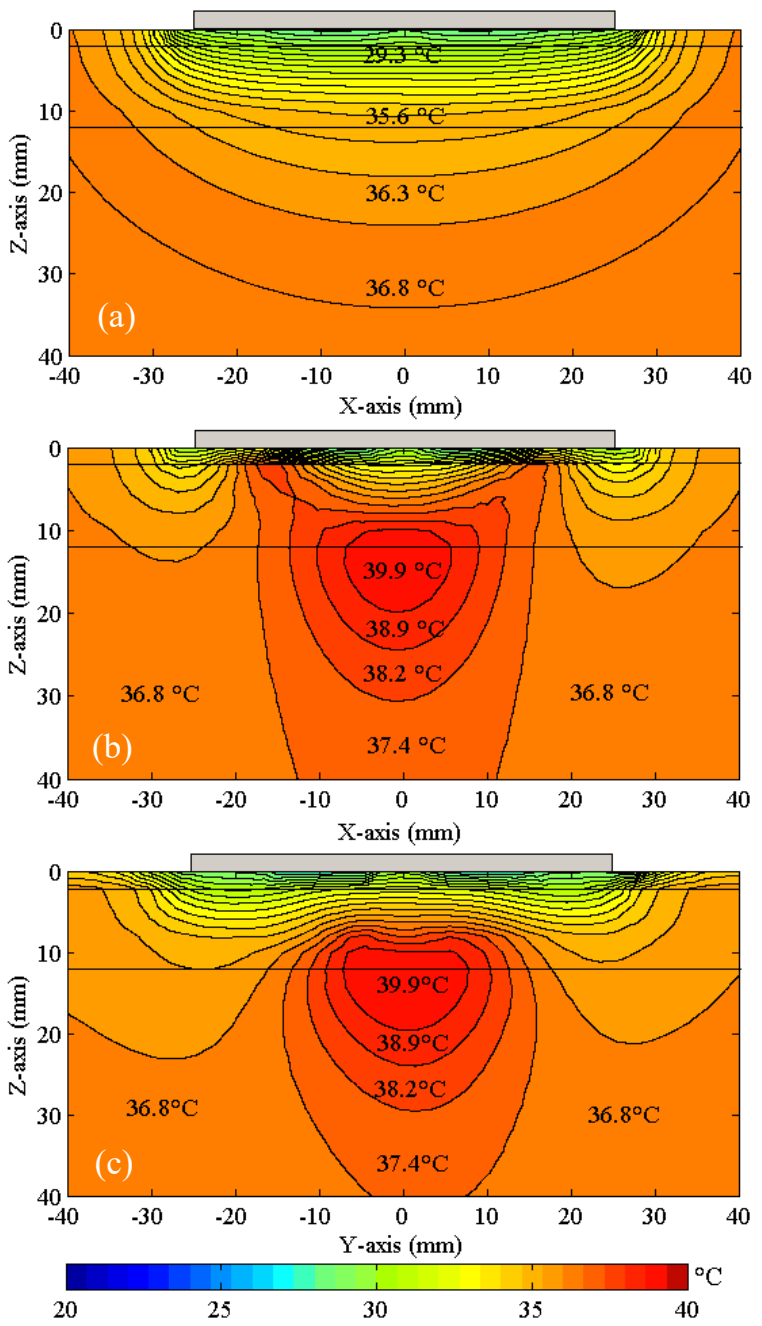

Fig. 4. Simulated thermal distribution in, (a) $x z$-plane before microwave is applied, (b) $x z$-plane with 5 minutes of microwave exposure, (c) $y z$ plane with 5 minutes of microwave exposure. 


\section{2) Thermal distribution}

To minimize the superficial hotspots, the microwave applicator was attached to a skin cooling system, which would activate when the detected temperature, based on the readings fed from the Doppler blood flow (LDF) optical monitor, exceeded a preset threshold value. Fig. 4(a) shows the thermal distribution of the calf model before the microwave heating was switched on $(t=0)$. On this occasion, the microwave applicator lowered the skin temperature from $36.7{ }^{\circ} \mathrm{C}$ to $29.3{ }^{\circ} \mathrm{C}$ because the applicator has high thermal conductivity and was exposed to a background temperature of $20^{\circ} \mathrm{C}$. The heat conduction had reached an equilibrium state and the surface of the skin and the applicator both had the same temperature of $29.3{ }^{\circ} \mathrm{C}$. At this point, the microwave applicator was switched on for 5 minutes and the thermal distributions in the $\mathrm{xz}-$ and $\mathrm{yz}-$ planes were shown in Fig. 4(b) and (c). It can be seen that the two superficial hotspots have now been mitigated and the skin temperature remained low at about $30^{\circ} \mathrm{C}$. A hotspot at $39.9^{\circ} \mathrm{C}$ can be found at about $15 \mathrm{~mm}$ below the skin surface in the muscle layer.

\section{RESULTS}

The results demonstrated below, are based on in vivo measurement on a human subject. Figure 5 shows the results in terms of flux which is a relative measure of the skin blood flow in an arbitrary unit measured by the LDF sensor. The skin temperature remained stable during microwave warming. The skin temperature varied within $1{ }^{\circ} \mathrm{C}$ over time, showing the effectiveness of the skin cooling system when the skin temperature exceeded the preset threshold temperature

Figure 6 shows the tissue oxygenation changes in the muscle in terms of the $\Delta \mathrm{HbO} 2 / \Delta \mathrm{HHb} / \Delta \mathrm{HbT}$ signals measured by the NIRS monitor. The initial 3 minutes of the baseline record was before the microwave switched on. There was some natural, spontaneous variation in tissue oxygenation signals this is typical of NIRS muscle measurement.

When the microwave was switched on, both the $\Delta \mathrm{HbO} 2$ and $\Delta \mathrm{HbT}$ signals, and to a lesser degree the $\Delta \mathrm{HHb}$ signal, went up. This increment shows that the microwave applicator successfully induced deep warming in the muscle, initiating vasodilation in the arterial blood vessels which carry more oxygenated blood (proportional to $\triangle \mathrm{HbO} 2$ ) than de-oxygenated blood (proportional to $\Delta \mathrm{HHb}$ ). After the applicator is switched off the signals begins to decrease as the dilation in the muscle is reduced.

\section{DISCUSSION AND CONCLUSION}

In the current work, we have incorporated a skin cooling system which can reduce superficial hotspots caused by microwave warming. As a result, the heat is mainly deposited in the muscle, and subsequently, the vasodilation and blood volume increase occur also predominantly in the muscle. This new design allows us to measure the thermal response in the muscle with minimal effect from the skin. While currently linear polarization has been used in the microwave applicator, a circular polarization that distributes energy more evenly on the skin surface would reduce superficial heat further. To this end, the feasibility of applying circular polarization to this application has been established in a recent simulation study [12]

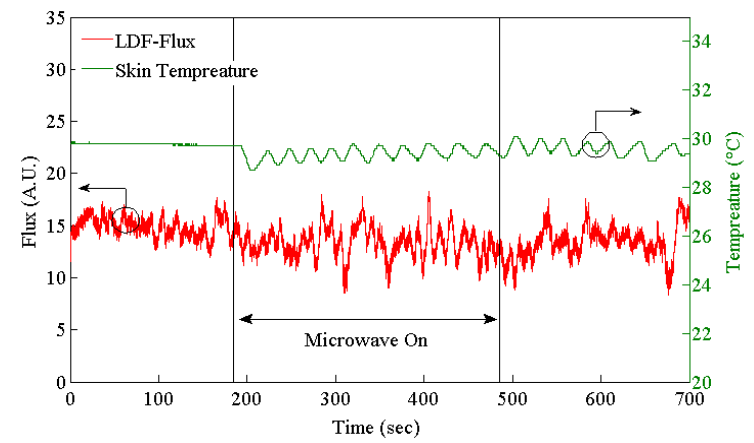

Fig.5 Measured flux and skin temperature with 5 minutes of microwave exposure:

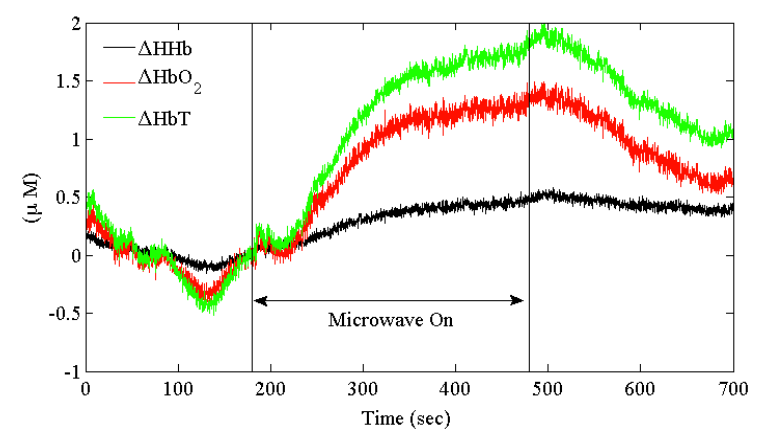

Fig. 6. Tissue oxygenation changes $(\Delta \mathrm{HbO} 2 / \Delta \mathrm{HHb} / \Delta \mathrm{HbT})$ over 5 minutes of microwave exposure

\section{REFERENCES}

[1] A. Rosen, "Applications of RF/microwaves in medicine," Microw. Theory Tech. IEEE. vol. 50, no. 3, pp. 963-974, 2002.

[2] P. Wust, B. Hildebrandt, and G. Sreenivasa, "Hyperthermia in combined treatment of cancer," Lancet Oncol., pp. 487-497, 2002

[3] A. Giombini, V. Giovannini, A. Di Cesare, P. Pacetti, N. IchinosekiSekine, M. Shiraishi, H. Naito, and N. Maffulli, "Hyperthermia induced by microwave diathermy in the management of muscle and tendon injuries.," Br. Med. Bull., vol. 83, pp. 379-96, Jan. 2007.

[4] A. Guy, "Therapeutic applications of electromagnetic power," Proc. IEEE, vol. 62, no. 1, pp. 55-75, 1974..

[5] C. K. Chou, "Evaluation of microwave hyperthermia applicators.," Bioelectromagnetics, vol. 13, no. 6, pp. 581-97, Jan. 1992.

[6] A. Guy, "Electromagnetic fields and relative heating patterns due to a rectangular aperture source in direct contact with bilayered biological tissue," Microw. Theory Tech. IEEE Trans., vol. 16, no. 2, pp. 214-223, 1971

[7] J. Lehmann, J. Sfonebridge, and A. Guy, “A comparison of patterns of stray radiation from therapeutic microwave applicators measured near tissue-substitute models and human subjects," Radio Sci., vol. 14, no. 6, pp. 271-283, 1979.

[8] J. Hand, J. Cheetham, and A. Hind, "Absorbed power distributions from coherent microwave arrays for localized hyperthermia," IEEE Trans. Microw. Theory Tech., vol. M, no. 5, pp. 484-489, 1986.

[9] M. Wolf, M. Ferrari, and V. Quaresima, "Progress of near-infrared spectroscopy and topography for brain and muscle clinical applications.," J. Biomed. Opt., vol. 12, no. 6, p. 062104, 2007.

[10] H. R. Underwood, a F. Peterson, and R. L. Magin, "Electric-field distribution near rectangular microstrip radiators for hyperthermia heating: theory versus experiment in water.," IEEE Trans. Biomed. Eng., vol. 39, no. 2, pp. 146-53, Feb. 1992.

[11] K. Wong, W. Chen, and W. Huang, "The absorption and coupling of an electromagnetic wave incident on a microstrip circuit with superstrate," Electromagn. Compat. IEEE Trans., vol. 34, no. 1, pp. $17-22,1992$

[12] A. Al-Armaghany, K. Tong, and T. S. Leung, "Superficial heat reduction technique for a hybrid microwave-optical device," in Engineering in Medicine and Biology Society (EMBC), 2013 35th Annual International Conference of the IEEE, 2013, pp. 3749-3752. 